\title{
A preliminary study of three-dimensional reconstruction of the human osseous labyrinth from micro-computed tomography scans
}

\author{
J. Skrzat ${ }^{1}$, A. Wróbel ${ }^{2}$, J. Walocha ${ }^{1}$ \\ ${ }^{1}$ Department of Anatomy, Jagiellonian University, Collegium Medicum, Cracow, Poland \\ ${ }^{2}$ Department of Medical Physics, Institute of Physics, Jagiellonian University, Cracow, Poland
}

[Received 12 August 2012; Accepted 3 November 2012]

\begin{abstract}
The adult human temporal bone was investigated using micro-computed tomography scans. The effects of the volume rendering used to create three-dimensional reconstructions were presented in images showing components of the osseous labyrinth. The three-dimensional reconstructions were performed to visualise the cochlea and the semicircular canals embedded in the petrous bone. The final product of this study was a digital three-dimensional model of the entire osseous labyrinth, which can be viewed at different angles on the computer screen. (Folia Morphol 2013; 72, 1: 17-21)
\end{abstract}

Key words: bony labyrinth, petrous bone, microtomography

\section{INTRODUCTION}

The osseous labyrinth is lodged inside the petrous portion of the temporal bone, also termed as the petrous bone. It consists of three parts: the vestibule, semicircular canals, and cochlea. The entire labyrinth is an intricate, irregular, spatial object of small size. These geometrical features force the application of sophisticated techniques to visualise its anatomy and topography. For a long time spiral computed tomography (CT) has been used to assess the morphology of the temporal bone and detect destructive processes in the middle and inner ear [9]. In recent years three-dimensional (3D) volume-rendered CT images have been used to demonstrate the anatomy of the temporal bone. The 3D multiplanar images helped to understand temporal bone anatomy and improved the ability to evaluate pathologic conditions $[1,2,3,8]$. However, microtomography seems to be the most adequate technique to illustrate the nuances of temporal bone anatomy. This modality allows the creation of cross-sections of a 3D-object with high resolution that may extend into the range of light microscopy, down to a few microns. Furthermore, the scans are used to recreate a virtual model which can be viewed at different angles and subjected to morphometric analysis. Application of the unified system for the spatial reconstruction and visualisation of the osseous labyrinth is helpful in objective and reproducible evaluation of its morphological diversity. Hereby, we tried to work out an effective way to reconstruct details of the inner structures of the petrous bone using micro-CT. The purpose of this study was to construct an accurate surface model of the osseous labyrinth and visualise the spatial orientation of the labyrinth components. This study did not contain any measurements of the osseous labyrinth because we focused on the methodology for 3D reconstruction and visualisation of the examined organ using computer software and micro-CT scans of the petrous bone. 


\section{MATERIALS AND METHODS}

The osseous material being the subject of the current study belongs to the Department of Anatomy of the Collegium Medicum of the Jagiellonian University. A single temporal bone was separated from a dry human skull, the morphological character of which indicated a young individual of female sex. The temporal bone subjected to the micro-CT showed no macroscopic defects and we did not observed any traces of infections of its anatomic regions. Information about previous diseases of the middle or inner ear is unavailable in the studied case because we used osteological material of unknown origin and history. This fact remains without meaning for the study, which is basically aimed at testing the capabilities of micro-CT and applied software to reconstruct and visualise the osseous labyrinth. Therefore, we used only one temporal bone in the study.

The separated temporal bone was cleaned of soil particles, washed with water, and immersed in $10 \%$ formaldehyde for 24 hours. Next, the bone was dried at room temperature. The petrous part of the temporal bone was cut off using an electric precision saw because the whole temporal bone was too big to be placed in the holder of the desktop microtomograph. Thus, the prepared sample was scanned with a micro-CT scanner (Skyscan 1172, N.V., Aartselaar, Belgium). The scanner was equipped with an X-ray detector: 11 Megapixel (4024 $\times 2680$ in total; $4000 \times 2400$ effective), 12-bit digital X-ray camera with $24 \times 36 \mathrm{~mm}$ field of view. The X-ray source voltage was set to $80 \mathrm{kV}$ and current to $100 \mu \mathrm{A}$. The resulting images had dimension $996 \times 900$ pixels, and the image pixel size was $27 \mu \mathrm{m}$. The projection images were acquired over an angular range of $180^{\circ}$ with an angular step of $0.5^{\circ}$. Projections were captured along the long axis of the petrous bone and reconstructed using NRECON ver. 1.6.5 SkyScan software based on the Feldkamp algorithm.

The 3D model of the osseous labyrinth was created in the program CT-analyser (CTAn). It is an application for deriving quantitative parameters and constructing visual models from scanned datasets obtained with SkyScan micro-CT instruments. The CTAn software allows real time volume rendered model viewing. The purpose of the model was to make visible the 3D structure of an object that has been imaged by micro-CT.

For viewing and manipulating the 3D surface rendered model generated from the micro-CT scans we also used a CT-volume (CTvol) application. Vol- ume rendering was used as a technique to display a 2D projection of a 3D, discretely sampled data set produced by the micro-CT scanner. Based on the histogram display of the intensities present in the volume data we adjusted interactively the transfer function that maps voxel intensities to provide the best perception of the observed structures. In that way we visualised different material components of the object having differing X-ray opacity. Visualisation of the air spaces was performed using the CTvox software because it allows for exploitation of the blending mode. By modifying the opacity we could control the visibility of the voxels.

The study was conducted with the approval (KBET/109/B/2012) of the Bioethics Committee of the Jagiellonian University.

\section{RESULTS}

The osseous labyrinth was reconstructed as a complex cavity consisting of the central vestibule, the semicircular canals, and the cochlea. The complex shape of the osseous labyrinth was presented as a 3D volume rendering object. Structures composing the osseous labyrinth were easily visible in the CT scans, and the 3D reconstruction was accepted as fully satisfactory. The performed surface and volume rendering allowed the generation of 3D models of the entire labyrinth or its particular components. The effects are presented in subsequent Figures 1-4.

The 3D visualisation based on interactive direct volume rendering gave an opportunity to observe the reconstructed structures at various angles. An important feature of visualisation was the ability to show anatomical structures embedded in bone. Thereby, we revealed the position of the labyrinth within the petrous bone (Figs. 1, 2). Surface reconstruction of the cochlea and the semicircular canals within the petrous bone was depicted in Figure 3.

All anatomical details (the cupula - apex of the cochlea, middle turn, and basal turn of the cochlea) of the external morphology of the cochlea were reconstructed (Fig. 3A). However, the vestibule in that projection is barely visible because it is embedded in the bone. In cantrast, the semicircular canals (anterior, lateral, posterior) were reconstructed as emerging from the bone (Fig. 3B). They are situated above and behind the vestibule. The semicircular canals are unequal in length, compressed from side to side, and each of them seems to describe a circle. Also, the common crus and 

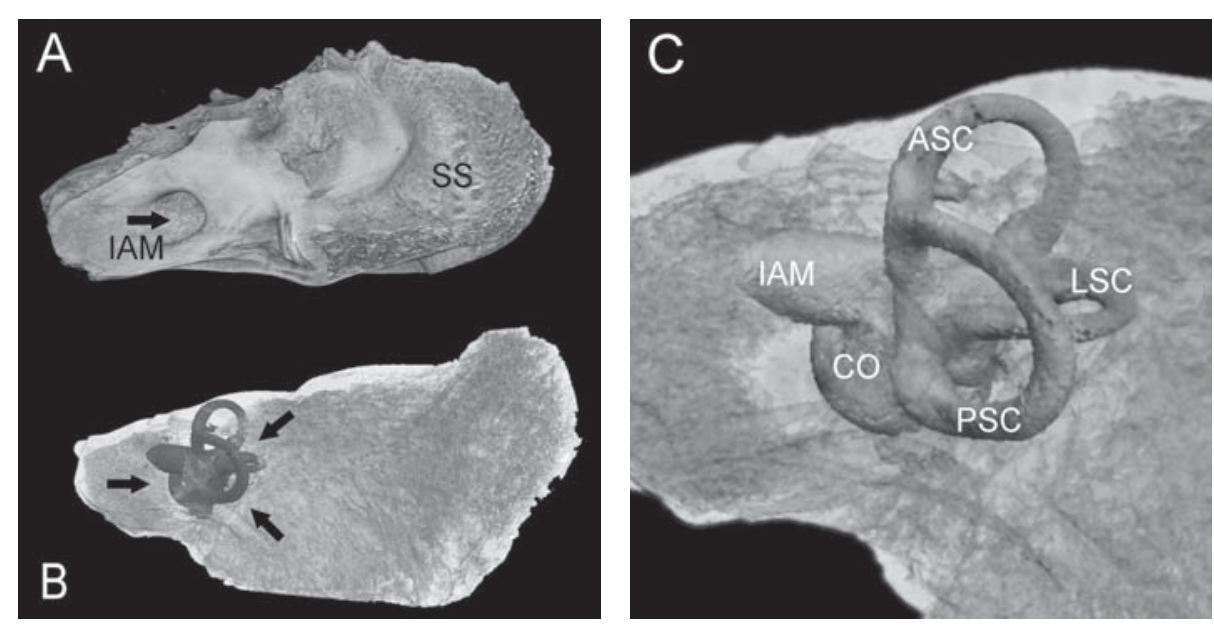

Figure 1. A. Volume reconstruction of the right petrous bone, visible medial surface; IAM — internal acoustic meatus; SS — sigmoid sulcus; B. The petrous bone is considered semi-transparent and the labyrinth is visible as an opaque object (indicated by arrows); C. Enlarged image of the osseous labyrinth in the postero-medial aspect; $\mathrm{CO}$ — cochlea; ASC — anterior semicircular canal; PSC — posterior semicircular canal; LSC — lateral semicircular canal.

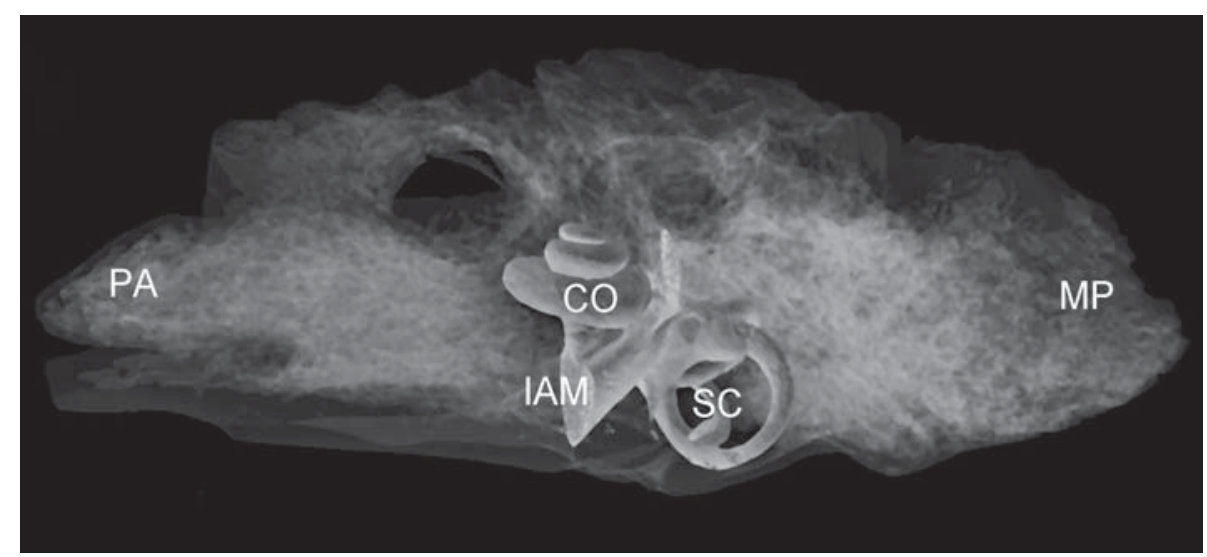

Figure 2. Superior view of the osseous labyrinth inside the petrous bone; PA — petrous apex; $\mathrm{CO}$ - cochlea; SC — semicircular canals; IAM - internal acoustic meatus; MP — mastoid part of the temporal bone (cerebellar surface).

the ampullas of each semicircular canal were reconstructed, and can be easily recognised in Figure 3B. The mutual topographical relationship between the cochlea and the semicircular canals is shown in Figure 4. The cochlea forms the anterior part of the labyrinth, whereas the semicircular canals are located posteriorly. Hence, Figure 4 presents the final effect of the digital reconstruction of the entire osseous labyrinth viewed in the anterior and posterior aspect.

The micro-CT examination of the temporal bone allowed a detailed imaging of the osseous labyrinth to be performed. The generated 3D model of the osseous labyrinth presents its overall appearance and may be used in comparative morphological studies. It also allows interactive exploration of the anatomy of the inner ear and helps to understand the spatial relationships within its components and surrounding structures.

\section{DISCUSSION}

The first morphological analyses of the inner structures of the temporal bone were based on manual sectioning. Histological sections only permitted 2D observations and planar measurements of the specimens [4]. However, that way of reconstruction was extremely time consuming and was rarely used in the anatomical research of the topography and morphology of the middle and inner ear [5].

Development of computers and graphic software enabled $3 D$ reconstructions to be carried out from the $2 \mathrm{D}$ images. The computer graphics system com- 

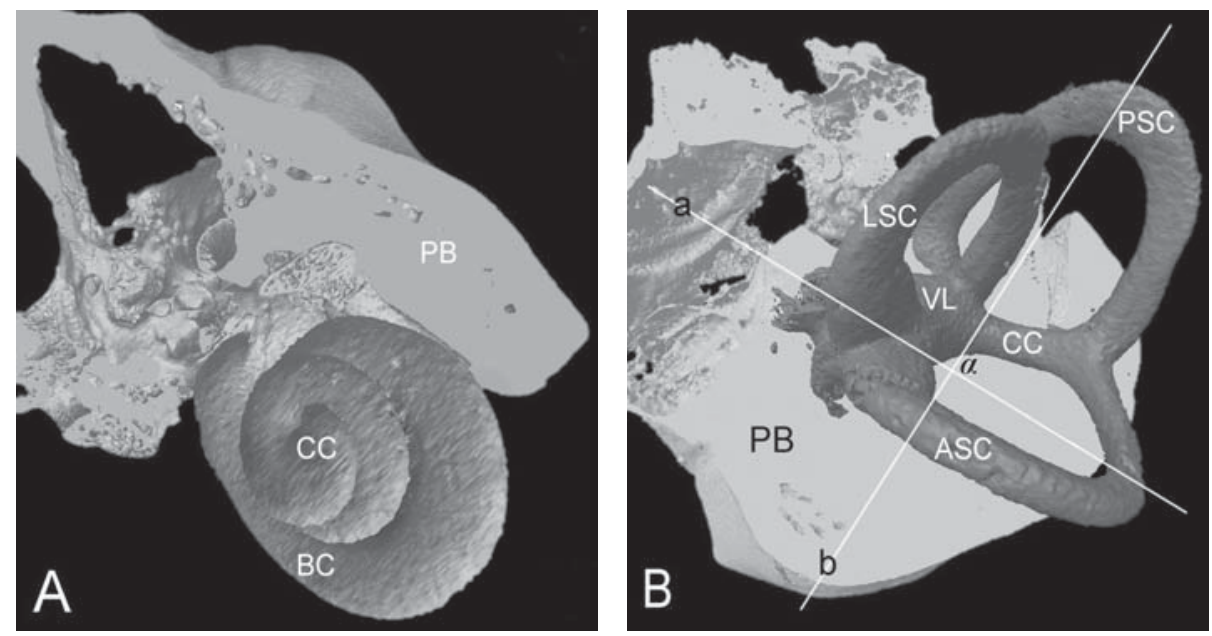

Figure 3. Surface reconstruction of the cochlea $(\mathbf{A})$ in the antero-inferior aspect, and the semicircular canals $(\mathbf{B})$ viewed from the posterior. Surrounding bone was partially removed; $\mathrm{PB}$ — petrous bone; $\mathrm{BC}$ — base of the cochlea; $\mathrm{VL}$ — vestibule; $\mathrm{ASC}$ — anterior semicircular canal; PSC — posterior semicircular canal; LSC — lateral semicircular canal; CC — common crus; a — axis of the plane of the anterior semicircular canal, $\mathrm{b}$ - axis of the plane of the posterior semicircular canal (long axis of the petrous bone), $\alpha \approx 90^{\circ}$.
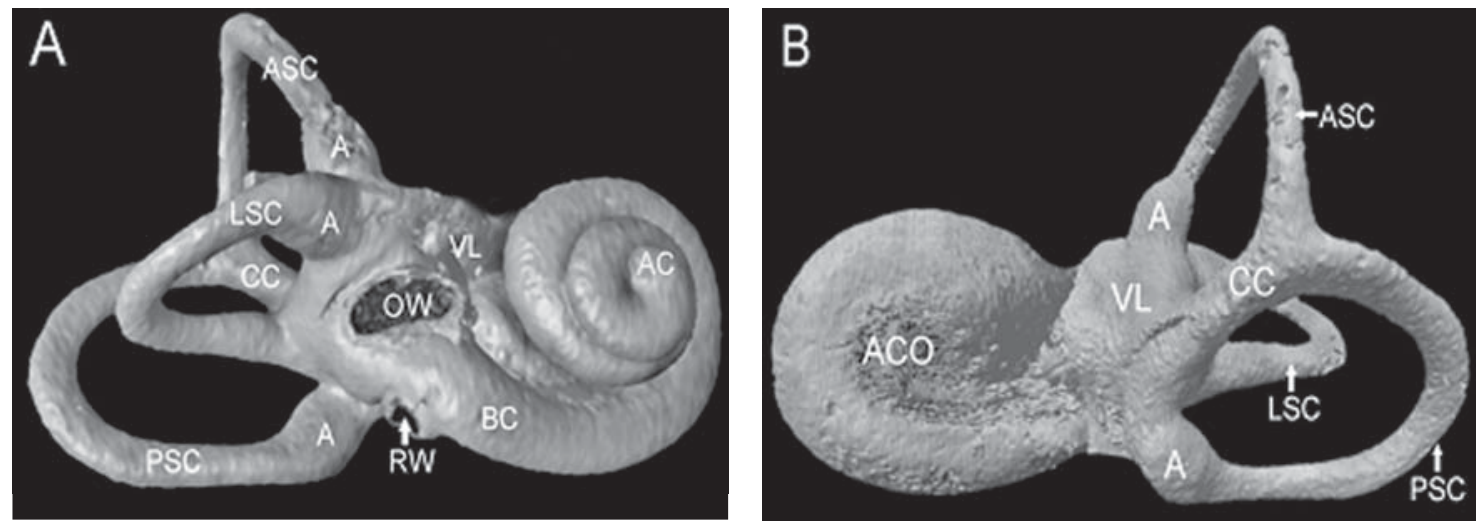

Figure 4. A digital model of the right osseous labyrinth; A. Anterolateral view; B. Posterosuperior view; BC — basal turn of the cochlea; $\mathrm{AC}$ — apex of the cochlea; VL — vestibule; $\mathrm{OW}$ — oval window; RW — round window; ACO — cochlear area; ASC — anterior semicircular canal; PSC — posterior semicircular canal; LSC — lateral semicircular canal; CC — common crus; A — ampulla.

bined with the histologic sections permitted the visualisation of the intratemporal bone structures in a 3D way, and viewing them from various angles [6].

The application of that novel modality in morphological science opened new cognitive horizons and deepened knowledge on the spatial organisation of the minute structures. Computer-based $3 \mathrm{D}$ models of the anatomical structures have become an important tool for learning and teaching anatomy. Three-dimensional computer-assisted reconstruction simplified the understanding of the complexity of the temporal bone and the relationship between its components $[7,11,13]$. In our case, the generated model of the osseous labyrinth seems to be helpful in understanding both its anatomy and topography within the temporal bone. Thus, it can be dedicated for educational purposes.

The current study was performed only on one temporal bone because our intention was to work out a convenient method for precise reconstruction and visualisation of the osseous labyrinth. Therefore we did not deal with morphometric and comparative analysis of the petrous bones and their inner structures. Although the study was limited to one sample it clearly showed that all components of the osseous labyrinth were reconstructed and displayed completely in high resolution. On the computer screen they can be viewed in 3D space at different angles. Performed reconstruction of the osseous labyrinth was based on 2000 micro-CT scans. The dimensions of each scan were $996 \times 900$ pixels, and the slice thick- 
ness was equal to $27.0 \mu \mathrm{m}$. This provided an extraordinary accuracy in modelling and approached a virtual appearance of the studied structures of the inner ear. Thereby, micro-CT is the best technique for anatomical study of any small osseous components. This modality is superior to the classical radiographic methods, which allow visualisation of the labyrinth components with lower resolution compared to micro-CT. Such images are blurred when magnified whereas the original scans are not always adequate to reveal all anatomical details of the inner structure of the petrous bone. In order to recognise the complex spatial relations of a labyrinth and its surrounding structures a clear visualisation is required. Precise imaging of the cochlea, the vestibule, and the semicircular canals is essential for comparative and quantitative studies. There is a little information on the morphological variation of the osseous labyrinth in man $[10,12]$. Possible reasons include accessibility to the micro-CT scanner and its technical capabilities. Another is the duration of the scanning process (as much as a few hours per sample), and limitations of the sample diameters (up to few centimetres). Therefore, the scanning of large objects (e.g. the whole skull) is impossible and the process would be extremely timeconsuming. For the time being, micro-CT provides images with the highest resolution and fidelity that can be obtained in various imaging modalities. The reconstructed structures reveal a large spectrum of detail which disappears in other radiographic techniques. The anatomical details of the temporal bone (e.g. labyrinth) are clearly delineated in the micro-CT scans. Therefore, a stack of images can be used to generate excellent computer models of the inner ear.

\section{ACKNOWLEDGEMENTS}

This research was carried out with equipment purchased thanks to the financial support of the European Regional Development Fund in the framework of the Polish Innovation Economy Operational Program (contract no. POIG.02.01.00-12-023/08).

\section{REFERENCES}

1. Bartling SH, Shukla V, Becker H, Brady TJ, Hayman A, Gupta R (2005) High-resolution flat-panel volume-CT of temporal bone. Part 1: axial preoperative anatomy. J Comput Assist Tomogr, 29: 420-423.

2. Dalchow CV, Weber AL, Yanagihara N, Bien S, Werner JA (2006) Digital volume tomography: radiologic examinations of the temporal bone. AJR, 186: 416-423.

3. Fatterpekar GM, Doshi AH, Dugar M, Delman BN, Naidich TP, Som PM (2006) Role of 3D CT in the evaluation of the temporal bone. Radiographics, 26 (suppl. 1): 117-132.

4. Green JD Jr, Marion MS, Erickson BJ, Robb RA, Hinojosa $R$ (1990) Three-dimensional reconstruction of the temporal bone. Laryngoscope, 100: 1-4.

5. Harada T, Ishii S, Tayama N (1988) Three-dimensional reconstruction of the temporal bone from histologic sections. Arch Otolaryngol Head Neck Surg, 114: 1139-1142.

6. Lutz C, Takagi A, Janecka IP, Sando I (1989) Three-dimensional computer reconstruction of a temporal bone. Otolaryngol Head Neck Surg, 101: 522-526.

7. Mason TP, Applebaum EL, Rasmussen M, Millman A, Evenhouse R, Panko W (2000) Virtual temporal bone: creation and application of a new computer based teaching tool. Otolaryngol Head Neck Surg, 122: 168-173.

8. Powitzky ES, Hayman LA, Bartling SH, Chau J, Gupta R, Shukla V (2006) High-resolution computed tomography of temporal bone. Part III: Axial postoperative anatomy. J Comput Assist Tomogr, 30: 337-343.

9. Shaffer M (1981) Computed tomography of the temporal bone. Radiographics, 1: 62-72.

10. Spoor F, Zonneveld F (1998) Comparative review of the human bony labyrinth. Am J Phys Anthropol, 41: 211-251 .

11. Tomandl BF, Hastreiter $P$, Eberhardt KEW, Rezk-Salama $C$, Naraghi R, Greess H, Nissen U, Huk WJ (2000) Virtual labyrinthoscopy: visualization of the inner ear with interactive direct volume rendering. Radiographics, 20: 547-558.

12. Tomura N, Sashi R, Kobayashi M, Hirano H, Hashimoto M, Watarai J (1995) Normal variations of the temporal bone on high-resolution CT: their incidence and clinical significance. Clin Radiol, 50: 144-148.

13. Wang H, Northrop C, Burgess B, Liberman MC, Merchant SN (2006) Three-dimensional virtual model of the human temporal bone: a stand-alone, downloadable teaching tool. Otol Neurotol, 27:452-457. 